\title{
OCORRÊNCIA DE AGENTES MICROBIOLÓGICOS EM FACHADAS DE EDIFICAÇÕES LOCALIZADAS EM SALVADOR/BA
}

\author{
CERQUEIRA, MILENA BORGES DOS SANTOS \\ Doutoranda em Engenharia Civil \\ Universidade Federal da Bahia \\ Bahia; Brasil \\ milena.bsc@gmail.com
}

\author{
BARBOSA, LUANNE BASTOS DE BRITTO \\ Mestranda em Engenharia Civil \\ Universidade Federal da Bahia \\ Bahia; Brasil \\ lubastosb@gmail.com
}

\section{COELHO, VINÍCIUS ALMEIDA}

Mestrando em Engenharia Civil

Universidade Federal da Bahia

Bahia; Brasil

mrvoelho@gmail.com

\author{
MARQUES, LUCAS SOUZA \\ Graduado em Engenharia Civil \\ Universidade Federal da Bahia \\ Bahia; Brasil \\ lucasmarqueseng@hotmail.com
}

\author{
MARTINS, MATHEUS GOMES \\ Mestrando em Engenharia Civil \\ Universidade Federal da Bahia \\ Bahia; Brasil \\ matheus.engmartins@gmail.com
}

\author{
SILVA, FRANCISCO GABRIEL \\ SANTOS \\ Professor Doutor \\ Universidade Federal da Bahia \\ Bahia; Brasil \\ fgabriel.ufba@gmail.com
}

\section{RESUMO}

Os agentes microbiológicos contribuem significativamente com a redução do desempenho das fachadas em edifícios brasileiros. Observa-se que o tipo de organismo e o seu padrão de desenvolvimento em fachadas ocorre de maneira diferenciada, podendo ser em função das características e constituição de cada material que compõem a fachada, dos detalhes construtivos presentes e das condições climáticas aos quais as fachadas estão expostas, por exemplo. Os revestimentos das fachadas com acabamento em pintura, localizados na cidade de Salvador/BA, apresentam uma frequente ocorrência de manchamento rosa. Diante disso, o presente trabalho tem como objetivo analisar a ocorrência das manifestações patológicas caracterizadas por manchas rosas que afetam os revestimentos e pinturas das fachadas das edificações localizadas em Salvador/BA. Para o alcance desse objetivo, a metodologia adotada foi aplicada em edificações localizadas em diferentes bairros da cidade, baseada em vistorias nas fachadas, realização de anamneses e mapeamento dos danos em revestimento com acabamento em pintura. $\mathrm{O}$ presente trabalho apresenta a relação entre características específicas das fachadas, tais como orientação cardeal, condições do entorno, e o desenvolvimento de manchas rosas.

Palavras-chave: fachada, patologia, manchamento, desempenho.

\section{ABSTRACT}

Microbiological agents contribute significantly to the reduction of façade performance in Brazilian buildings. It is observed that the type of organism and its pattern of development in façades occurs in a different way, depending on the characteristics and constitution of each material that makes up the façade, the present constructive details and the climatic conditions to which the façades are located. exposed, for example. Facade coatings with paint finishes, located in the city of Salvador / BA, have a frequent occurrence of pink staining. Given this, the present work aims to analyze the occurrence of pathological manifestations characterized by pink spots that affect the cladding and painting of the facades of buildings located in Salvador / BA. To achieve this goal, the methodology adopted was applied to buildings located in different neighborhoods of the city, based on façade surveys, anamneses and damage mapping on paint finishes. This paper presents the relationship between specific façade characteristics, such as cardinal orientation, surrounding conditions, and the development of pink spots.

Keywords: facade, pathology, staining, performance. 


\section{INTRODUÇÃO}

Segundo a ABNT NBR 15575-4:2013 a fachada é componente do sistema de vedação vertical interno e externo, pois é parte da edificação habitacional que limita verticalmente a edificação e seus ambientes.

As fachadas apresentam grande variedade e variabilidade nos materiais, estes exibem propriedades físico-químicas e mecânicas diferentes, resultando em desempenhos distintos. Tipos de cimentos, tintas, argamassas, revestimento, dentre outros elementos diferentes, são postos em conjunto e estes precisam apresentar comportamento satisfatório, simultaneamente, para garantir a durabilidade do sistema como um todo.

A degradação das fachadas das edificações é iniciada tão logo a construção é concluída por interferência da ação de alguns agentes, tais como: como umidade, insolação, vento e biodeteriorização.

A degradação ao longo do tempo ocorre na medida em que o material perde a capacidade de responder às exigências, ao longo do tempo, aos agentes de deterioração, à natureza do material e ainda da própria idade do material, culminando com o surgimento das patologias (GASPAR; BRITO et al., 2005).

Os diversos fatores ambientais que colaboram com a degradação do sistema de revestimento das fachadas de edificações podem ocorrer com intensidades diferenciadas em função da região de influência, ou seja, do microclima do edifício, e mais especificamente, em função das orientações das fachadas. Sendo, portanto, necessária uma análise minuciosa desses efeitos para a obtenção de um adequado diagnóstico das degradações nos edifícios (NASCIMENTO, 2016).

Dentre os diversos tipos de manifestações patológicas presentes em revestimento de fachadas, o aparecimento de microrganismos biológicos tem se destacado em fachadas de edifícios localizados em Salvador/BA, e em parte deles, manchas rosas tem tomada grandes proporções.

O surgimento de microrganismos biológicos se dá devido à permanência prolongada de água em fachadas com menor exposição ao sol, dependendo da presença de nutrientes (FLORES-COLEN, 2009).

MORTON e SURMAN (1997) definem biodeterioração como o estudo da deterioração dos materiais de importância econômica pela atividade biológica vital dos organismos. Manchas de vegetação parasitária são caracterizadas pela presença de organismos biológicos, que são as algas, líquenes, musgos e outras plantas superiores de pequeno arbóreo (ex.: trepadeiras), na presença de luz e umidade (FLORES-COLEN, 2009).

As manchas rosas identificadas em fachadas com acabamento em pintura ou textura têm intrigado os moradores e profissionais da construção civil, por gerar grande impacto visual para todos que observam a fachada. Diante do apresentado, julga-se importante estudar o que pode influenciar na aparição destas manifestações patológicas nas edificações, assim como sua influência no desempenho dos sistemas de revestimentos de fachadas.

O presente trabalho tem como objetivo analisar ocorrência de manchas rosas nas fachadas, realizando a catalogação de algumas edificações localizadas em Salvador/BA com o dano citado.

\subsection{Biodeterioração em fachadas de edificações}

A fachada das edificações está sujeita à diversas condições de exposição, e desta forma, está propícia ao surgimento de biodeterioração. Os microorganismos envolvidos no processo de biodeterioração se adaptam aos mais diversos substratos, devido, principalmente, à diversidade microbiana que compõe o biofilme (SANTOS, 2011).

A biodeterioração segundo HUECK (1968), pode ser classificada por:

- Processo mecânico: o material resulta degradado pela atividade do organismo como seu deslocamento ou crescimento;

- Assimilação química: forma mais frequente, quando um material é degradado em função de suas propriedades nutritivas, como a degradação da celulose, por exemplo;

- Descarte: quando ocorre uma avaria pela produção e excreção de metabolitos, que pode corroer ou pigmentar o material;

- Esfarinhamento: é a forma de biodeterioração que ocorre quando a simples presença de um microrganismo, ou de seus excrementos, o torna inaceitável. 
A biodeterioração mais evidente, causada por algas e cianobactérias, está ligada à estética, causando manchas não só em rochas, como também em materiais cimentícios, na madeira e superfícies pintadas. O requisito básico para que ocorra esse tipo de biodeterioração é simplesmente haver luz suficiente para seu desenvolvimento. O aparecimento dessas manchas, de coloração variada e crescimento rápido, pode ser em tons de verde, rosa ou marrons, que dão à edificação uma aparência de desleixo (GAYLARDE; GAYLARDE, 2000, ALLSOPP, 2004).

Os problemas advindos do processo de biodeterioração têm uma ocorrência ampla no mundo e alcançam níveis mais preocupantes em locais de clima tropical, como o Brasil, onde as condições de temperatura, umidade e luminosidade são extremamente favoráveis ao crescimento da maioria dos microrganismos que atuam no processo (TIANO, 1998).

\section{METODOLOGIA}

No presente trabalho foi realizado um mapeamento em Salvador/BA para identificar edificações que apresentam fachadas com manchamentos de cor rosa em seu acabamento. Foram identificados e levantados dados de 6 edifícios localizados em diferentes bairros da cidade (Rio Vermelho, Federação, Paralela e Horto Bela Vista), no período de julho a outubro de 2018 .

Foi confeccionado um termo de consentimento para a administração do condomínio, solicitando a autorização para entrada no mesmo, coleta de dados e registro fotográfico. Após a assinatura do termo pelo gestor do edifício, foi realizada a visita para realização de registros fotográficos, onde foi utilizada câmera fotográfica Canon EOS Rebel T6 e um questionário para esclarecimento e coleta de informações indispensáveis para o presente trabalho, como por exemplo a idade da edificação, tempo da última pintura e tipo de tinta que foi utilizada para pintura das fachadas.

Todas as orientações das fachadas das edificações foram fotografadas e analisadas. A partir da finalização da coleta dos dados necessários, foi realizada a quantificação das áreas manchadas e calculadas as porcentagens das áreas manchadas através da delimitação das áreas.

\section{RESULTADOS E DISCUSSÕES}

A Figura 1 apresenta a localização das edificações mapeadas no presente estudo, na cidade de Salvador/BA. Para o presente trabalho, foram coletados dados de 6 edificações.

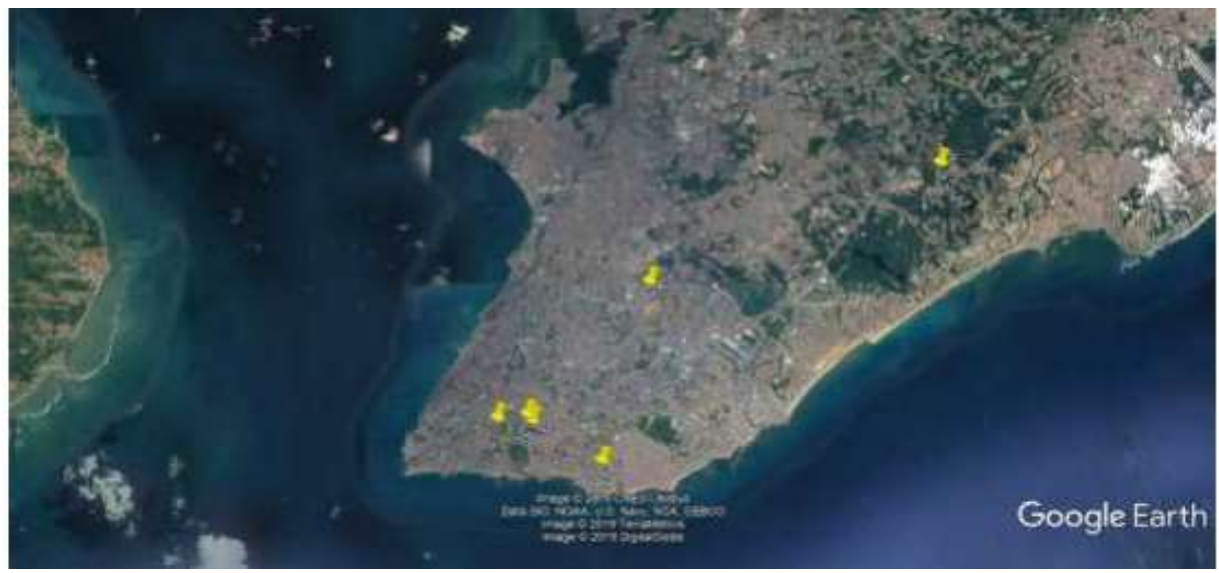

Figura 1: Localização das edificações analisadas (GOOGLE EARTH, 2019)

Foram escolhodas as edificações que apresentaram manchamento rosa em pelo menos uma de suas fachadas. As figuras 2 à 7 apresentam imagens das fachadas com manchas rosas, assim como as características de cada edificação. 


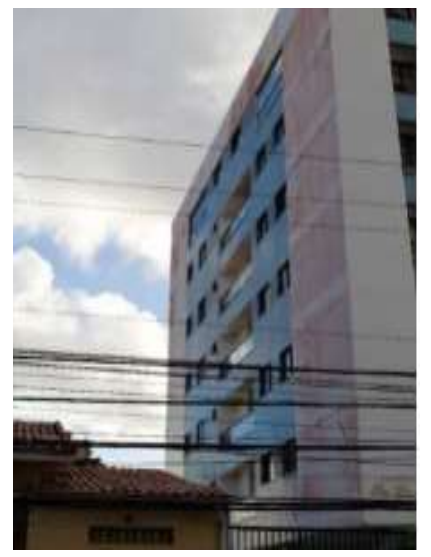

\begin{tabular}{|c|c|}
\hline \multicolumn{2}{|c|}{ Edificação A } \\
\hline Tipo de uso & Residencial \\
\hline Idade & 4 anos \\
\hline Número de pavimentos & 6 pavimentos \\
\hline Acabamento de fachada & Pintura \\
\hline Tipo da Pintura/Textura & Acrílica \\
\hline Última pintura & 2017 \\
\hline
\end{tabular}

Figura 2: Edificação A: Fachada Sul com Manchas e apresentação de dados coletados (AUTORES, 2019)

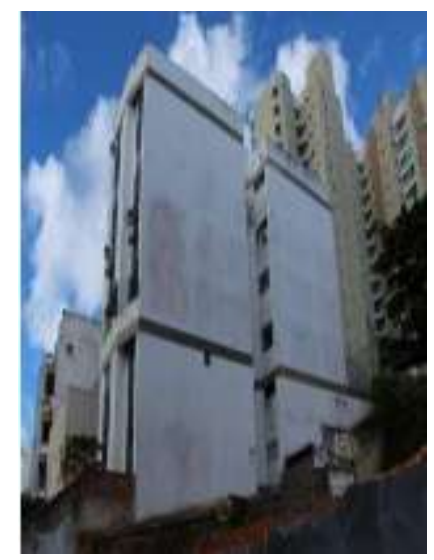

\begin{tabular}{|c|c|}
\hline \multicolumn{2}{|c|}{ Edificação B } \\
\hline Tipo de uso & Residencial \\
\hline Idade & 34 anos \\
\hline Número de pavimentos & 7 pavimentos \\
\hline Acabamento de fachada & Pintura \\
\hline Tipo da Pintura/Textura & Acrílica \\
\hline Última pintura & 2006 \\
\hline
\end{tabular}

Figura 3: Edificação B: Fachada Noroeste com Manchas e apresentação de dados coletados (AUTORES, 2019)

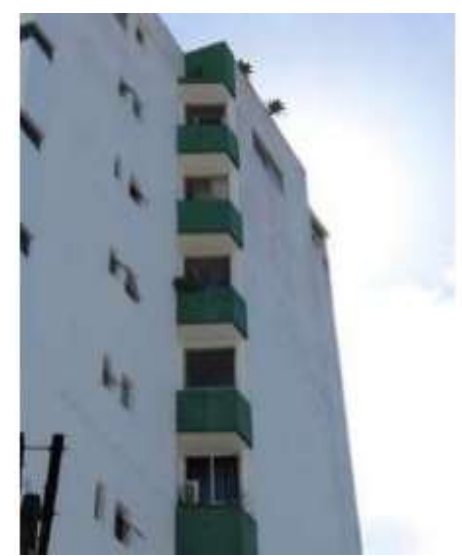

\begin{tabular}{|c|c|}
\hline \multicolumn{2}{|c|}{ Edificação C } \\
\hline Tipo de uso & Residencial \\
\hline Idade & 35 anos \\
\hline Número de pavimentos & 9 pavimentos \\
\hline Acabamento de fachada & Pintura \\
\hline Tipo da Pintura/Textura & Acrílica \\
\hline Última pintura & 2016 \\
\hline
\end{tabular}

Figura 4: Edificação C: Fachada Sudeste com Manchas e apresentação de dados coletados (AUTORES, 2019) 


\section{СВРAT 2020

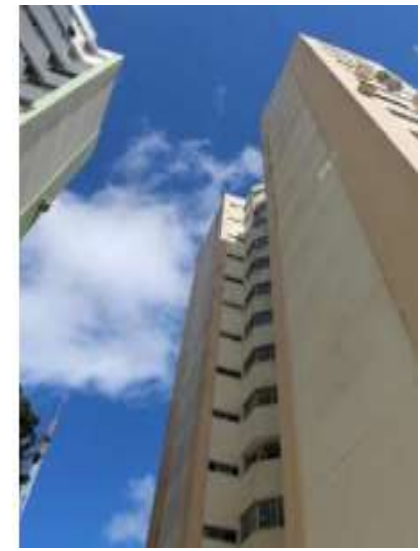

\begin{tabular}{|c|c|}
\hline \multicolumn{2}{|c|}{ Edificação D } \\
\hline Tipo de uso & Residencial \\
\hline Idade & 30 anos \\
\hline Número de pavimentos & 13 pavimentos \\
\hline Acabamento de fachada & Textura \\
\hline Tipo da Pintura/Textura & Acrílica \\
\hline Última pintura & 2015 \\
\hline
\end{tabular}

Figura 5: Edificação D: Fachada Sudeste com Manchas e apresentação de dados coletados (AUTORES, 2019)

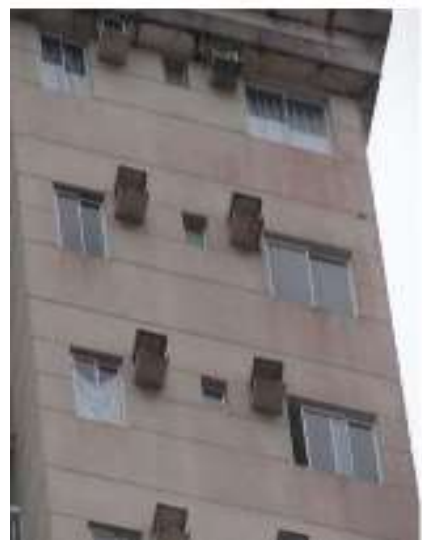

\begin{tabular}{|c|c|}
\hline \multicolumn{2}{|c|}{ Edificação E } \\
\hline Tipo de uso & Residencial \\
\hline Idade & 6 anos \\
\hline Número de pavimentos & 25 pavimentos \\
\hline Acabamento de fachada & Textura \\
\hline Tipo da Pintura/Textura & Acrílica \\
\hline Última pintura & 2016 \\
\hline
\end{tabular}

Figura 6: Edificação E: Fachada Sul com Manchas e apresentação de dados coletados (AUTORES, 2019)

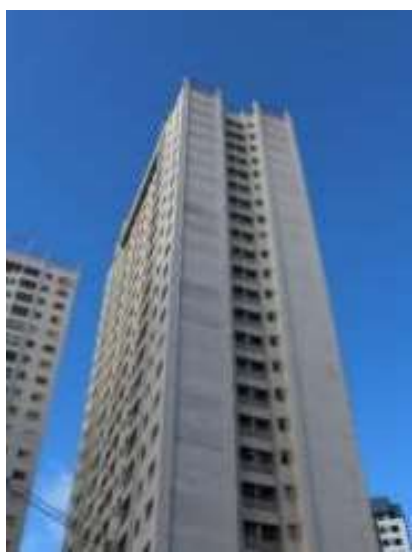

\begin{tabular}{|c|c|}
\hline \multicolumn{2}{|c|}{ Edificação F } \\
\hline Tipo de uso & Residencial \\
\hline Idade & 5 anos \\
\hline Número de pavimentos & 24 pavimentos \\
\hline Acabamento de fachada & Textura \\
\hline Tipo da Pintura/Textura & Acrílica \\
\hline Última pintura & 2013 \\
\hline
\end{tabular}

Figura 7: Edificação F: Fachada Sul com Manchas e apresentação de dados coletados (AUTORES, 2019)

As fachadas A, B e C apresentam acabamento de fachada em textura, enquanto as fachadas das edificações D, E e F apresentam acabamento em pintura.

É importante ressaltar que as fotos 2 à 7 apresentam as fachadas com maior área de manchamento em relação à todas as fachadas de cada edifício.

Nas vistorias foi realizada a quantificação da área com presença de manchas rosas em relação à área total das fachadas, para identificar a porcentagem de mancha de cada edificação. Os resultados calculados estão apresentados no Quadro 1. 
Quadro 1 - Porcentagem de Manchas rosas nas edificações analisadas

\begin{tabular}{|c|c|}
\hline Edifício & $\begin{array}{c}\text { Porcentagem de mancha em toda } \\
\text { a fachada }\end{array}$ \\
\hline A & $39 \%$ \\
\hline B & $20 \%$ \\
\hline C & $11 \%$ \\
\hline D & $31 \%$ \\
\hline E & $19 \%$ \\
\hline F & $34 \%$ \\
\hline
\end{tabular}

Diante dos dados apresentados, observa-se que a edificação A apresenta a maior porcentagem de mancha em relação à área total das fachadas, apresentando um valor de 39\%. O que condiz com o observado visualmente durante a vistoria, em que as fachadas apresentam uma estética marcada pelo manchamento rosa, se destacando em relação à área restante de cor branca. A edificação A é a edificação mais nova dentre as analisadas, porém apresentou maior incidência de manchas.

A Edificação C é a edificação de maior idade, 35 anos, entre as analisadas, ao mesmo tempo que apresenta menor índice de manchamento em relação à sua área total de fachadas. É possível verificar, a partir da análise percentual apresentada, que a maior incidência de manchas não está relacionada diretamente com maior idade. Sendo necessário verificar outras condições que interferem no desempenho da fachada e na sua vulnerabildade à biodeerioração.

O período em que as fachadas passaram por manutenções mais recentes também não apresentam relação direta com a incidência de manchamento. A edificação A, de maior incidência, passou por manutenção mais recente entre as apresentadas, ano de 2017, assim como a de menor incidência, edificação C, também passou por manutenções mais recentes, ano de 2016.

A Figura 8 apresenta a porcentagem de manchas em relação às orientações cardeais das 6 edificações analisadas.

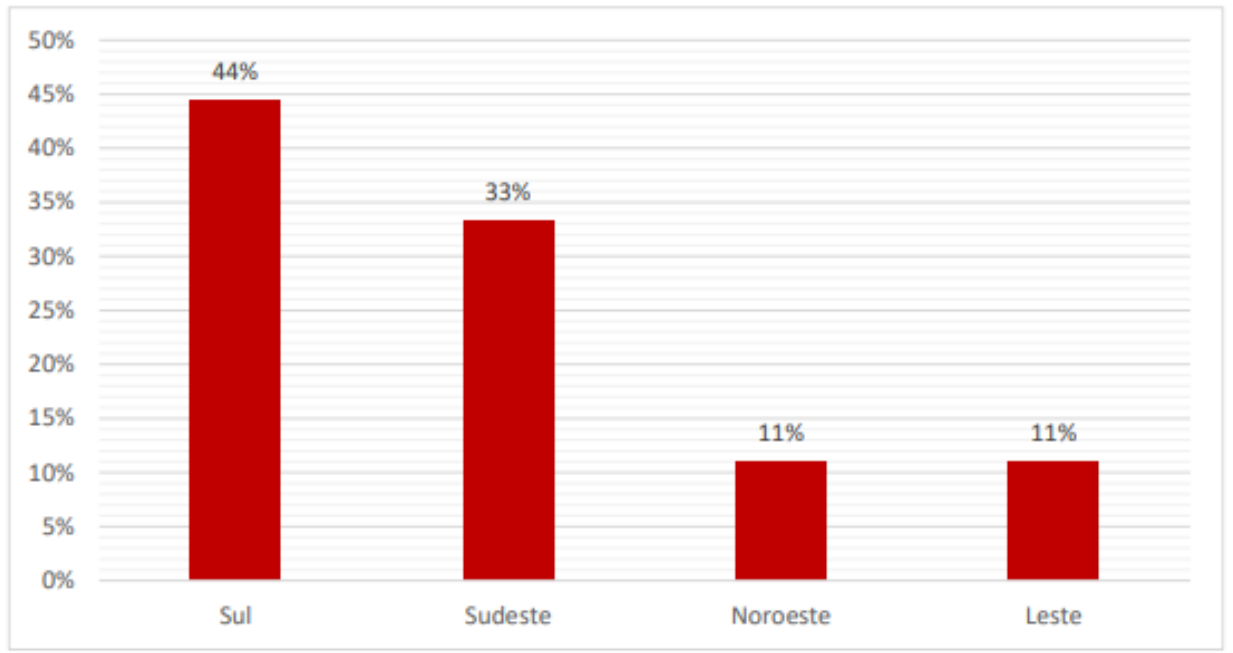

Figura 8: Relação entre Porcentagem de manchas e orientação cardeal das fachadas (AUTORES, 2019)

Dentre as edificações analisadas, a orientação cardeal Sul apresenta a incidência de $44 \%$ de manchas em relação à sua área total seguida da orientação cardeal Sudeste com 33\%, e Noroeste e Leste com 11\%.

Diante do exposto não foi possível verificar a relação da orientação cardeal sul e a maior incidência de danos, em relação às demais orientações cardeais. Sendo necessário investigar mais fatores que atuam diretamente no processo de degradação das fachadas, pois apesar das edificações analisadas estarem localizadas na mesma cidade, o micro-clima interfere de forma diferente em cada uma delas. E essas condições, tais como circulação do vento e incidência solar, vão variar de acordo com as condições do entorno específicas de cada uma. 
É importante destacar que a realização de pinturas nas fachadas para a retirada da mancha não é eficiente, se a causa do seu surgimento não for identificada e eliminada. Isso foi possível verificar, já que as manchas voltaram em alguns meses após a execução de uma nova pintura.

\section{CONCLUSÕES}

Diante do estudo realizado foi possível verificar que diferentes orientações cardeais das fachadas de diferentes edificações apresentaram manchas rosas. Tendo a orientação Sul apresentado maior índice dessa manifestação patológicas, dentre as edificações analisadas.

Não foi possível estabelecer relação entre a orientação cardeal, idade e última pintura, com o surgimento das manchas. Tendo como explicação, as peculiaridades de uso que cada edificação apresenta, suas condições de vizinhança e técnicas e materiais utilizados no período da execução. Tornando-se evidente o quanto importante é aprofundar os estudos nesta área, no intuito de colaborar com um desempenho adequado das fachadas das edificações ao longo de sua vida útil.

A realização de análise microbiológica é necessária para identificação das espécies de microorganismos causadores das manchas, para que desta forma seja compreendido o processo de degradação ocasionado pelos mesmos, assim como para a indicação das medidas de manutenção eficientes, já que foi verificada que a realização de manutenções recentes nas fachadas não evitou a sua manifestação precoce.

\section{AGRADECIMENTOS}

Agradecemos à Fundação de Amparo à Pesquisa do Estado da Bahia (FAPESB) pelo apoio fincanceiro e incentivo à pesquisa.

\section{REFERÊNCIAS}

ABNT - ASSOCIAÇÃO BRASILEIRA DE NORMAS TÉCNICAS. NBR 15575-4: Edificações Habitacionais Desempenho Parte 4: Requisitos para os sistemas de vedações verticais internas e externas. Rio de Janeiro, 2013.

ALLSOPP, D.; SEAL, K. J.; GAYLARD, C. C. Introduction to iodeterioration. 2nd. ed. Cambridge, USA: Cambridge University Press, 2004.

FLORES-COLEN, I. Metodologia de avaliação do desempenho em serviço de fachadas rebocadas na óptica da manutenção predictiva. Tese (Doutorado). Engenharia Civil do Instituto Superior Técnico. Universidade Técnica de Lisboa, Lisboa, 2009.

GASPAR, P. L.; BRITO, J.. Assessment of the overall degradation level of an element, based on field data. 10DBMC International Conférence On Durability of Building Materials and Components. Lyon, França, 2005.

GAYLARDE, P.M.; GAYLARDE, C.C. Algae and cyanobacteria onpainted buildings in Latin America. International Biodeterioration \& Biodegradation, v.46, Issue 2, p.93-97, Sept. 2000.

HUECK, H. J. The Biodeterioration of materials as a part of hylobiology. Central Laboratorium TNO, Delft, The Netherlands, 1968.

MORTON, L. H. G.; SURMAN, B. S. The role of biofilms in biodeterioration - A review. In:WORKSHOP DURABILIDADE DAS CONSTRUÇÕES. São Leopoldo: UNISINOS/ ANTAC, 1997.

NASCIMENTO, M. L. M. Aplicação de simulação higrotérmica na investigação da degradação de fachadas de edifícios. Dissertação de mestrado, Universidade de Brasília, 2016. 


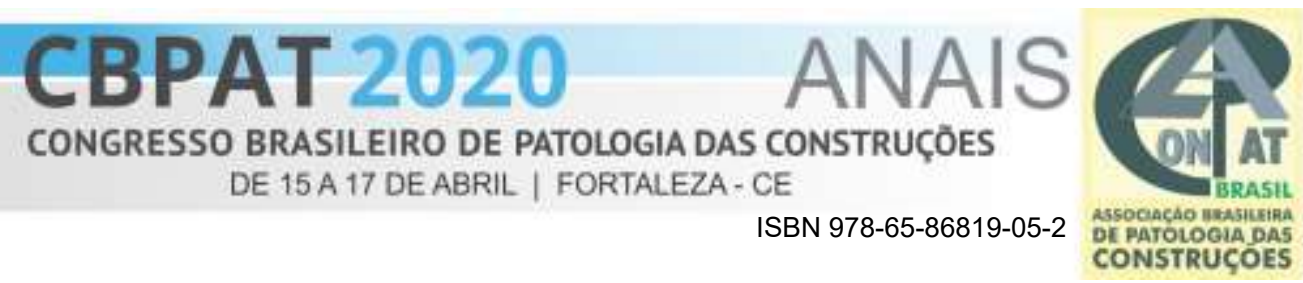

SANTOS, A. B. DOS. Identificação biológica e aplicação de biocidas na população microbiana que forma os biofilmes de alguns monumentos históricos da Bahia. Dissertação de Mestrado, Universidade Federal da Bahia, Faculdade de Arquitetura. 2011.

TIANO, P. Biodeterioration of monumental rocks: decay mechanisms and control methods. Science and Technology for Cultural Heritage. 1998. 